\title{
Rhodium-Catalyzed Asymmetric Synthesis of Indanones: Development of a New "Axially-Chiral" Bisphosphine Ligand
}

Ryo Shintani, Keiji Yashio, Tomoaki Nakamura, Kazuhiro Okamoto, Toyoshi Shimada, and Tamio Hayashi*

Department of Chemistry, Graduate School of Science, Kyoto University, Sakyo, Kyoto 606-8502, Japan

\section{Supporting Information}

\section{General}

All air- and moisture-sensitive manipulations were carried out with standard Schlenk techniques under nitrogen or in a glove box under argon.

THF was either distilled over benzophenone ketyl under nitrogen or purified by passing through a neutral alumina column under nitrogen. $\mathrm{Et}_{2} \mathrm{O}$ was purified by passing through a neutral alumina column under nitrogen. $\mathrm{CH}_{2} \mathrm{Cl}_{2}$ was distilled over $\mathrm{CaH}_{2}$ under nitrogen. DMF was distilled over $\mathrm{CaH}_{2}$ under vacuum. DME was distilled over benzophenone ketyl under nitrogen.

1-Phenyl-2-propyn-1-ol (Wako Chemicals), 2-propyn-1-ol (Nacalai Tesque), 3,4dihydro-2H-pyran (Wako Chemicals), chloro(ethyl)dimethylsilane (Fluoro Chem.), methyl trifluoromethanesulfonate (Kanto Chemicals), tetrabutylammonium fluoride (Aldrich; 1.0 M solution in THF), iodine (Nacalai Tesque), $p$-toluenesulfonic acid (Nacalai Tesque; monohydrate), pyridinium chlorochromate (Aldrich), $\mathrm{Cu}$ powder (Acros), $\mathrm{LiAlH}_{4}$ (Wako Chemicals), $n$-BuLi (Kanto Chemicals; $1.58 \mathrm{M}$ solution in hexane), and $t$-BuLi (Kanto Chemicals; $1.45 \mathrm{M}$ solution in pentane) were used as received.

1-Phenyl-3-triethylsilyl-2-propyn-1-ol (1a), ${ }^{1}$ 1-phenyl-3-trimethylsilyl-2-propyn-1ol (1c) ${ }^{1} \quad(R)$-2-diphenylphosphinyl-2'-methoxy-1,1'-binaphthyl $((R)-4),{ }^{2}$ and

\footnotetext{
${ }^{1}$ Shintani, R.; Okamoto, K.; Hayashi, T. J. Am. Chem. Soc. 2005, 127, 2872.

${ }^{2}$ Uozumi, Y.; Tanahashi, A.; Lee, S.-Y.; Hayashi, T. J. Org. Chem. 1993, 58, 1945.
} 
$\left[\mathrm{Rh}(\mathrm{cod})_{2}\right] \mathrm{BF}_{4}{ }^{3}$ were synthesized following the literature procedures. $(R)$ - and (S)-1phenyl-2-propyn-1-ol were obtained by enzymatic kinetic resolution of the racemate, following the literature procedure. ${ }^{4}$

All other chemicals and solvents were purchased from Aldrich, Wako Chemicals, TCI, or Kanto Chemicals and used as received.

\section{Synthesis of Substrates}

The yields have not been optimized.

\section{1-Phenyl-3-dimethyl(ethyl)silyl-2-propyn-1-ol (1b)}<smiles>CCCSC#CC(O)c1ccccc1</smiles>

$1 \mathrm{~b}$

Two drops of $\mathrm{HCl}$ (12 M aqueous) were added to a mixture of 1-phenyl-2propyn-1-ol (2.43 mL, $20.0 \mathrm{mmol})$ and 3,4-dihydro-2H-pyran (2.01 mL, $22.0 \mathrm{mmol})$, and this was stirred for $2 \mathrm{~h}$ at room temperature. The reaction was quenched with $\mathrm{Na}_{2} \mathrm{CO}_{3}$ and the solid was removed by filtration with $\mathrm{Et}_{2} \mathrm{O}$. The volatiles were removed under vacuum to afford a pale yellow oil. This oil was dissolved in THF $(60 \mathrm{~mL})$ and $n$-BuLi (12.3 mL, $19.4 \mathrm{mmol} ; 1.58 \mathrm{M}$ solution in hexane) was added to it at $-40{ }^{\circ} \mathrm{C}$. After stirring for $30 \mathrm{~min}$ at $-40{ }^{\circ} \mathrm{C}$, chloro(ethyl)dimethylsilane $(2.74 \mathrm{~mL}$, $19.5 \mathrm{mmol}$ ) was added, and the resulting mixture was stirred for $30 \mathrm{~min}$ at $-40{ }^{\circ} \mathrm{C}$ and for $2 \mathrm{~h}$ at room temperature. The reaction was quenched with water and extracted with hexane. The organic layer was dried over $\mathrm{MgSO}_{4}$, filtered, and concentrated under vacuum to afford a pale yellow oil. This oil was dissolved in $\mathrm{MeOH}(80 \mathrm{~mL})$ and $p$-toluenesulfonic acid (95.1 mg, $0.500 \mathrm{mmol}$; monohydrate) was then added to it. The resulting solution was stirred for $12 \mathrm{~h}$ at room temperature and the reaction was quenched with $\mathrm{NaHCO}_{3}$. After removal of $\mathrm{MeOH}$ under vacuum, the residue was poured into $\mathrm{NaHCO}_{3}$ (aqueous, saturated) and extracted with $\mathrm{Et}_{2} \mathrm{O}$. The organic layer was dried over $\mathrm{MgSO}_{4}$, filtered, and concentrated under vacuum. The residue was chromatographed on silica gel with $\mathrm{Et}_{2} \mathrm{O} /$ hexane =

${ }^{3}$ Schenck, T. G.; Downes, J. M.; Milne, C. R. C.; MacKenzie, P. B.; Boucher, H.; Whelan, J.; Bosnich, B. Inorg. Chem. 1985, 24, 2334.

${ }^{4}$ Raminelli, C.; Comasseto, J. V.; Andrade, L. H.; Porto, A. L. M. Tetrahedron: Asymmetry 2004, 15, 3117. 
$1 / 3$ to afford compound $\mathbf{1 b}$ as a pale yellow oil ( $3.59 \mathrm{~g}, 16.4 \mathrm{mmol} ; 85 \%$ yield).

${ }^{1} \mathrm{H} \mathrm{NMR}\left(\mathrm{CDCl}_{3}\right): \delta 7.55\left(\mathrm{~d},{ }^{3} J_{\mathrm{HH}}=7.4 \mathrm{~Hz}, 2 \mathrm{H}\right), 7.38\left(\mathrm{t},{ }^{3} J_{\mathrm{HH}}=6.9 \mathrm{~Hz}, 2 \mathrm{H}\right), 7.33(\mathrm{t}$, $\left.{ }^{3} J_{\mathrm{HH}}=7.2 \mathrm{~Hz}, 1 \mathrm{H}\right), 5.46\left(\mathrm{~d},{ }^{3} J_{\mathrm{HH}}=6.3 \mathrm{~Hz}, 1 \mathrm{H}\right), 2.16\left(\mathrm{~d},{ }^{3} J_{\mathrm{HH}}=6.3 \mathrm{~Hz}, 1 \mathrm{H}\right), 1.01\left(\mathrm{t},{ }^{3} J_{\mathrm{HH}}\right.$ $=7.8 \mathrm{~Hz}, 3 \mathrm{H}), 0.63\left(\mathrm{q},{ }^{3} \mathrm{~J}_{\mathrm{HH}}=7.8 \mathrm{~Hz}, 2 \mathrm{H}\right), 0.17(\mathrm{~s}, 6 \mathrm{H}) .{ }^{13} \mathrm{C} \mathrm{NMR}\left(\mathrm{CDCl}_{3}\right): \delta$ 140.4, 128.6, 128.4, 126.7, 105.3, 90.8, 65.0, 7.9, 7.3, -2.4. Anal. Calcd for $\mathrm{C}_{13} \mathrm{H}_{18} \mathrm{OSi}$ : C, 71.50; $\mathrm{H}, 8.31$. Found: $\mathrm{C}, 71.42 ; \mathrm{H}, 8.22$.

$(R)-\mathbf{1 b}(98 \%$ ee) and $(S)-\mathbf{1 b}$ (98\% ee) were synthesized in the same way starting from (S)-1-phenyl-2-propyn-1-ol and ( $R$-1-phenyl-2-propyn-1-ol, respectively. $(R)$ 1b: $[\alpha]^{20}{ }_{D}+21.2\left(c 1.00, \mathrm{CHCl}_{3}\right)$. (S)-1b: $[\alpha]^{20}{ }_{\mathrm{D}}-21.5$ (c 1.05, $\left.\mathrm{CHCl}_{3}\right)$.

\section{3-Dimethyl(ethyl)silyl-2-propynal (CAS 3111-95-3)}

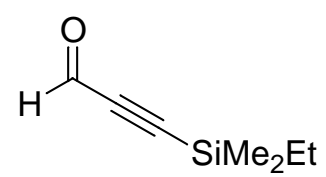

A solution of 3-dimethyl(ethyl)silyl-2-propyn-1-ol (2.85 g, $20.0 \mathrm{mmol}$; prepared in the same way as $\mathbf{1 b}$, starting from 2-propyn-1-ol) in $\mathrm{CH}_{2} \mathrm{Cl}_{2}(25 \mathrm{~mL})$ was added to a mixture of pyridinium chlorochromate $(6.47 \mathrm{~g}, 30.0 \mathrm{mmol})$ and $\mathrm{NaOAc}(492 \mathrm{mg}, 6.00$ $\mathrm{mmol})$ in $\mathrm{CH}_{2} \mathrm{Cl}_{2}(25 \mathrm{~mL})$, and the resulting mixture was stirred for $3 \mathrm{~h}$ at room temperature. The reaction mixture was diluted with $\mathrm{Et}_{2} \mathrm{O}(50 \mathrm{~mL})$ and filtered through celite and then through a pad of florisil. The solvent was removed under vacuum at $0{ }^{\circ} \mathrm{C}$, and the residue was chromatographed on silica gel with $\mathrm{Et}_{2} \mathrm{O} /$ pentane $=1 / 4$ to afford 3-dimethyl(ethyl)silyl-2-propynal as a colorless oil (2.05 g, $14.6 \mathrm{mmol} ; 73 \%$ yield).

${ }^{1} \mathrm{H} \mathrm{NMR}\left(\mathrm{CDCl}_{3}\right): \delta 9.16(\mathrm{~s}, 1 \mathrm{H}), 1.00\left(\mathrm{t},{ }^{3} J_{\mathrm{HH}}=7.7 \mathrm{~Hz}, 3 \mathrm{H}\right), 0.67\left(\mathrm{q},{ }^{3} J_{\mathrm{HH}}=7.9 \mathrm{~Hz}\right.$, $2 \mathrm{H}), 0.21(\mathrm{~s}, 6 \mathrm{H}) .{ }^{13} \mathrm{C} \mathrm{NMR}\left(\mathrm{CDCl}_{3}\right): \delta 176.6,102.6,102.4,7.2,7.0,-3.1$.

\section{1-(p-Tolyl)-3-dimethyl(ethyl)silyl-2-propyn-1-ol (1d)}

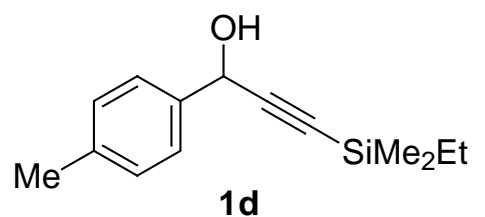

p-Tolylmagnesium bromide (4.48 $\mathrm{mL}, 3.60 \mathrm{mmol}$; $0.80 \mathrm{M}$ solution in THF) was added dropwise over $1 \mathrm{~h}$ to a solution of 3-dimethyl(ethyl)silyl-2-propynal (421 mg, $3.00 \mathrm{mmol})$ in THF $(5.0 \mathrm{~mL})$ at $-20{ }^{\circ} \mathrm{C}$. The mixture was stirred for $1 \mathrm{~h}$ at $-20{ }^{\circ} \mathrm{C}$ and 
for $2 \mathrm{~h}$ at room temperature, and the reaction was quenched with $\mathrm{HCl}(10 \%$ aqueous). After extraction with $\mathrm{Et}_{2} \mathrm{O}$, the organic layer was dried over $\mathrm{MgSO}_{4}$, filtered, and concentrated under vacuum. The residue was chromatographed on silica gel with $\mathrm{Et}_{2} \mathrm{O} /$ hexane $=1 / 3$ to afford compound $\mathbf{1 d}$ as a colorless oil $(467 \mathbf{m g}$, $2.01 \mathrm{mmol} ; 67 \%$ yield).

${ }^{1} \mathrm{H}$ NMR $\left(\mathrm{CDCl}_{3}\right): \delta 7.44\left(\mathrm{~d},{ }^{3} J_{\mathrm{HH}}=8.1 \mathrm{~Hz}, 2 \mathrm{H}\right), 7.19\left(\mathrm{~d},{ }^{3} J_{\mathrm{HH}}=8.1 \mathrm{~Hz}, 2 \mathrm{H}\right), 5.42(\mathrm{~d}$, $\left.{ }^{3} J_{\mathrm{HH}}=6.3 \mathrm{~Hz}, 1 \mathrm{H}\right), 2.36(\mathrm{~s}, 3 \mathrm{H}), 2.06\left(\mathrm{~d},{ }^{3} J_{\mathrm{HH}}=6.5 \mathrm{~Hz}, 1 \mathrm{H}\right), 1.00\left(\mathrm{t},{ }^{3} J_{\mathrm{HH}}=7.9 \mathrm{~Hz}, 3 \mathrm{H}\right)$, $0.62\left(\mathrm{q},{ }^{3} J_{\mathrm{HH}}=7.9 \mathrm{~Hz}, 2 \mathrm{H}\right), 0.17(\mathrm{~s}, 6 \mathrm{H}) .{ }^{13} \mathrm{C} \mathrm{NMR}\left(\mathrm{CDCl}_{3}\right): \delta 138.2,137.6,129.3,126.7$, 105.5, 90.5, 64.9, 21.1, 7.9, 7.3, -2.4. Anal. Calcd for $\mathrm{C}_{14} \mathrm{H}_{20} \mathrm{OSi}$ : C, 72.36; H, 8.67. Found: $\mathrm{C}, 72.43 ; \mathrm{H}, 8.62$.

\section{1-(p-Anisyl)-3-dimethyl(ethyl)silyl-2-propyn-1-ol (1e)}<smiles>CCOC#CC(O)c1ccc(OC)cc1</smiles>

This was synthesized from $p$-anisylmagnesium bromide, following the procedure for compound 1d. Pale yellow oil, $82 \%$ yield.

${ }^{1} \mathrm{H} \mathrm{NMR}\left(\mathrm{CDCl}_{3}\right): \delta 7.47\left(\mathrm{~d},{ }^{3} J_{\mathrm{HH}}=8.8 \mathrm{~Hz}, 2 \mathrm{H}\right), 6.91\left(\mathrm{~d},{ }^{3} J_{\mathrm{HH}}=8.7 \mathrm{~Hz}, 2 \mathrm{H}\right), 5.41(\mathrm{~d}$, $\left.{ }^{3} J_{\mathrm{HH}}=6.2 \mathrm{~Hz}, 1 \mathrm{H}\right), 3.82(\mathrm{~s}, 3 \mathrm{H}), 2.05\left(\mathrm{~d},{ }^{3} J_{\mathrm{HH}}=6.2 \mathrm{~Hz}, 1 \mathrm{H}\right), 1.01\left(\mathrm{t},{ }^{3} J_{\mathrm{HH}}=7.8 \mathrm{~Hz}, 3 \mathrm{H}\right)$, $0.63\left(\mathrm{q},{ }^{3} J_{\mathrm{HH}}=7.8 \mathrm{~Hz}, 2 \mathrm{H}\right), 0.17(\mathrm{~s}, 6 \mathrm{H}) .{ }^{13} \mathrm{C} \mathrm{NMR}\left(\mathrm{CDCl}_{3}\right): \delta 159.7,132.7,128.2,113.9$ 105.6, 90.5, 64.6, 55.3, 7.9, 7.3, -2.4. Anal. Calcd for $\mathrm{C}_{14} \mathrm{H}_{20} \mathrm{O}_{2} \mathrm{Si}$ : C, 67.70; H, 8.12. Found: C, 67.85; H, 8.07.

\section{1-(m-Tolyl)-3-dimethyl(ethyl)silyl-2-propyn-1-ol (1f)}<smiles>CCOC#CC(O)c1cccc(C)c1</smiles>

This was synthesized from $m$-tolylmagnesium bromide, following the procedure for compound 1d. Colorless oil, $84 \%$ yield.

${ }^{1} \mathrm{H} \mathrm{NMR}\left(\mathrm{CDCl}_{3}\right): \delta 7.37(\mathrm{~s}, 1 \mathrm{H}), 7.35\left(\mathrm{~d},{ }^{3} J_{\mathrm{HH}}=7.6 \mathrm{~Hz}, 1 \mathrm{H}\right), 7.27\left(\mathrm{t},{ }^{3} J_{\mathrm{HH}}=7.5 \mathrm{~Hz}\right.$, $1 \mathrm{H}), 7.14\left(\mathrm{~d},{ }^{3} J_{\mathrm{HH}}=7.4 \mathrm{~Hz}, 1 \mathrm{H}\right), 5.42\left(\mathrm{~d},{ }^{3} J_{\mathrm{HH}}=6.2 \mathrm{~Hz}, 1 \mathrm{H}\right), 2.37(\mathrm{~s}, 3 \mathrm{H}), 2.09\left(\mathrm{~d},{ }^{3} J_{\mathrm{HH}}=\right.$ $6.2 \mathrm{~Hz}, 1 \mathrm{H}), 1.01\left(\mathrm{t},{ }^{3} J_{\mathrm{HH}}=8.0 \mathrm{~Hz}, 3 \mathrm{H}\right), 0.63\left(\mathrm{q},{ }^{3} \mathrm{~J}_{\mathrm{HH}}=7.9 \mathrm{~Hz}, 2 \mathrm{H}\right), 0.17(\mathrm{~s}, 6 \mathrm{H}) .{ }^{13} \mathrm{C}$ 
$\operatorname{NMR}\left(\mathrm{CDCl}_{3}\right): \delta 140.3,138.3,129.1,128.5,127.5,123.8,105.5,90.7,65.1,21.4,7.9,7.2$, -2.4. Anal. Calcd for $\mathrm{C}_{14} \mathrm{H}_{20} \mathrm{OSi}$ : C, 72.36; H, 8.67. Found: $\mathrm{C}, 72.07 ; \mathrm{H}, 8.66$.

\section{1-(3,4-Dimethylphenyl)-3-dimethyl(ethyl)silyl-2-propyn-1-ol (1g)}<smiles>CCSC#CC(O)c1ccc(C)c(C)c1</smiles>

This was synthesized from 3,4-dimethylphenylmagnesium bromide, following the procedure for compound $\mathbf{1 d}$. Colorless oil, 78\% yield.

${ }^{1} \mathrm{H}$ NMR $\left(\mathrm{CDCl}_{3}\right): \delta 7.33-7.25(\mathrm{~m}, 2 \mathrm{H}), 7.14\left(\mathrm{~d},{ }^{3} J_{\mathrm{HH}}=7.7 \mathrm{~Hz}, 1 \mathrm{H}\right), 5.39\left(\mathrm{~d},{ }^{3} \mathrm{~J}_{\mathrm{HH}}=\right.$ $6.1 \mathrm{~Hz}, 1 \mathrm{H}), 2.28(\mathrm{~s}, 3 \mathrm{H}), 2.26(\mathrm{~s}, 3 \mathrm{H}), 2.10\left(\mathrm{~d},{ }^{3} J_{\mathrm{HH}}=6.2 \mathrm{~Hz}, 1 \mathrm{H}\right), 1.01\left(\mathrm{t},{ }^{3} J_{\mathrm{HH}}=8.0 \mathrm{~Hz}\right.$, $3 \mathrm{H}), 0.63\left(\mathrm{q},{ }^{3} \mathrm{~J}_{\mathrm{HH}}=7.8 \mathrm{~Hz}, 2 \mathrm{H}\right), 0.17(\mathrm{~s}, 6 \mathrm{H}) .{ }^{13} \mathrm{C} \mathrm{NMR}\left(\mathrm{CDCl}_{3}\right): \delta 137.9,136.71$, 136.69, 129.7, 128.0, 124.1, 105.8, 90.2, 64.8, 19.7, 19.4, 7.9, 7.2, -2.4. HRMS (ESI) calcd for $\mathrm{C}_{15} \mathrm{H}_{22} \mathrm{OSiNa}\left(\mathrm{M}+\mathrm{Na}^{+}\right)$269.1332, found 269.1333.

\section{1-(3,4-Methylenedioxyphenyl)-3-dimethyl(ethyl)silyl-2-propyn-1-ol (1h)}

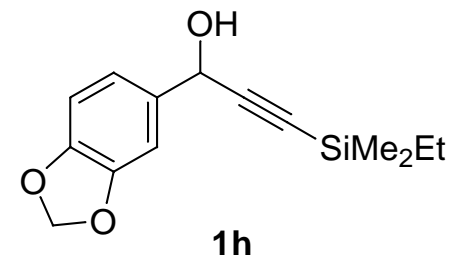

This was synthesized from 3,4-methylenedioxyphenylmagnesium bromide, following the procedure for compound $1 \mathrm{~h}$. Pale yellow oil, 68\% yield.

${ }^{1} \mathrm{H}$ NMR $\left(\mathrm{CDCl}_{3}\right): \delta 7.05(\mathrm{~s}, 1 \mathrm{H}), 7.01\left(\mathrm{~d},{ }^{3} J_{\mathrm{HH}}=8.1 \mathrm{~Hz}, 1 \mathrm{H}\right), 6.79\left(\mathrm{~d},{ }^{3} J_{\mathrm{HH}}=7.9 \mathrm{~Hz}\right.$, $1 \mathrm{H}), 5.97(\mathrm{~s}, 2 \mathrm{H}), 5.36\left(\mathrm{~d},{ }^{3} J_{\mathrm{HH}}=5.7 \mathrm{~Hz}, 1 \mathrm{H}\right), 2.14\left(\mathrm{~d},{ }^{3} J_{\mathrm{HH}}=5.5 \mathrm{~Hz}, 1 \mathrm{H}\right), 1.00\left(\mathrm{t},{ }^{3} J_{\mathrm{HH}}=\right.$ $7.8 \mathrm{~Hz}, 3 \mathrm{H}), 0.63\left(\mathrm{q},{ }^{3} \mathrm{~J}_{\mathrm{HH}}=7.9 \mathrm{~Hz}, 2 \mathrm{H}\right), 0.17(\mathrm{~s}, 6 \mathrm{H}) .{ }^{13} \mathrm{C} \mathrm{NMR}\left(\mathrm{CDCl}_{3}\right): \delta 147.7,147.5$, 134.5, 120.4, 108.0, 107.4, 105.4, 101.1, 90.4, 64.6, 7.8, 7.2, -2.5. HRMS (ESI) calcd for $\mathrm{C}_{14} \mathrm{H}_{18} \mathrm{O}_{3} \mathrm{SiNa}\left(\mathrm{M}+\mathrm{Na}^{+}\right)$285.0917, found 285.0918. 


\section{Synthesis of Ligand $(R, R)-3$}

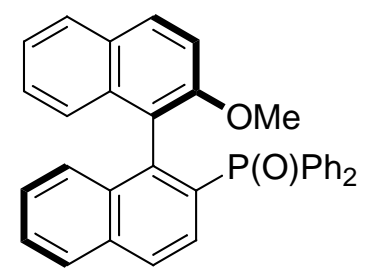

$(R)-4$

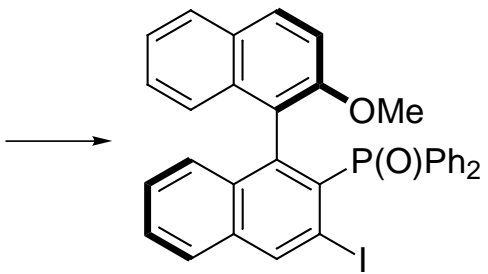

$(R)-5$

t-BuLi (10.3 mL, $15.0 \mathrm{mmol} ; 1.45 \mathrm{M}$ solution in pentane) was added to a solution of $(R)-4(1.45 \mathrm{~g}, 2.99 \mathrm{mmol})$ in THF $(150 \mathrm{~mL})$ at $-96{ }^{\circ} \mathrm{C}$ and the mixture was stirred for $2 \mathrm{~min}$. A solution of iodine $(2.70 \mathrm{~g}, 10.6 \mathrm{mmol})$ in THF $(10 \mathrm{~mL})$ was then added to it, and the reaction mixture was warmed to room temperature over $10 \mathrm{~min}$. After addition of $\mathrm{Na}_{2} \mathrm{SO}_{3}$ (10\% aqueous), this was extracted with EtOAc and the organic layer was washed with $\mathrm{NaHCO}_{3}$ (aqueous, saturated), and then with $\mathrm{NaCl}$ (aqueous, saturated). The organic layer was dried over $\mathrm{MgSO}_{4}$, filtered, and concentrated under vacuum. The residue was chromatographed on silica gel with $\mathrm{C}_{6} \mathrm{H}_{6} /$ EtOAc $=$ $1 / 1$ to afford $(R)-5$ as a brown solid (931 $\mathrm{mg}, 1.53 \mathrm{mmol} ; 51 \%$ yield).

${ }^{1} \mathrm{H}$ NMR $\left(\mathrm{CDCl}_{3}\right): \delta 8.80\left(\mathrm{~d},{ }^{4} J_{\mathrm{HH}}=2.5 \mathrm{~Hz}, 1 \mathrm{H}\right), 7.75\left(\mathrm{~d},{ }^{3} J_{\mathrm{HH}}=8.2 \mathrm{~Hz}, 1 \mathrm{H}\right), 7.59-$ $7.52(\mathrm{~m}, 3 \mathrm{H}), 7.48\left(\mathrm{t},{ }^{3} \mathrm{~J}_{\mathrm{HH}}=7.5 \mathrm{~Hz}, 1 \mathrm{H}\right), 7.46\left(\mathrm{~d},{ }^{3} J_{\mathrm{HH}}=9.2 \mathrm{~Hz}, 1 \mathrm{H}\right), 7.38\left(\mathrm{td},{ }^{3} J_{\mathrm{HH}}=7.5\right.$ $\mathrm{Hz}$ and $\left.{ }^{4} J_{\mathrm{HH}}=1.4 \mathrm{~Hz}, 1 \mathrm{H}\right), 7.27-7.20(\mathrm{~m}, 4 \mathrm{H}), 7.15\left(\mathrm{t},{ }^{3} J_{\mathrm{HH}}=7.8 \mathrm{~Hz}, 1 \mathrm{H}\right), 7.06-6.97(\mathrm{~m}$, $3 \mathrm{H}), 6.93\left(\mathrm{td},{ }^{3} J_{\mathrm{HH}}=7.5 \mathrm{~Hz}\right.$ and $\left.{ }^{4} J_{\mathrm{HH}}=1.4 \mathrm{~Hz}, 1 \mathrm{H}\right), 6.84\left(\mathrm{~d},{ }^{3} J_{\mathrm{HH}}=9.2 \mathrm{~Hz}, 1 \mathrm{H}\right), 6.75(\mathrm{~d}$, $\left.{ }^{3} J_{\mathrm{HH}}=8.3 \mathrm{~Hz}, 1 \mathrm{H}\right), 6.73\left(\mathrm{td},{ }^{3} \mathrm{~J}_{\mathrm{HH}}=8.0 \mathrm{~Hz}\right.$ and $\left.{ }^{4} J_{\mathrm{HH}}=3.2 \mathrm{~Hz}, 2 \mathrm{H}\right), 3.67(\mathrm{~s}, 3 \mathrm{H}) .{ }^{13} \mathrm{C}$ $\operatorname{NMR}\left(\mathrm{CDCl}_{3}\right): \delta 154.2,143.1\left(\mathrm{~d}, J_{\mathrm{CP}}=9.3 \mathrm{~Hz}\right), 142.3\left(\mathrm{~d}, J_{\mathrm{CP}}=9.8 \mathrm{~Hz}\right), 135.5\left(\mathrm{~d}, J_{\mathrm{CP}}=2.1\right.$ $\mathrm{Hz}), 134.2,133.9\left(\mathrm{~d}, J_{\mathrm{CP}}=105.1 \mathrm{~Hz}\right), 133.2\left(\mathrm{~d}, J_{\mathrm{CP}}=107.8 \mathrm{~Hz}\right), 132.2\left(\mathrm{~d}, J_{\mathrm{CP}}=10.9 \mathrm{~Hz}\right)$, $131.8\left(\mathrm{~d}, J_{\mathrm{CP}}=9.8 \mathrm{~Hz}\right), 131.2,131.11,131.09\left(\mathrm{~d}, J_{\mathrm{CP}}=10.3 \mathrm{~Hz}\right), 131.09,130.8\left(\mathrm{~d}, J_{\mathrm{CP}}=\right.$ $107.0 \mathrm{~Hz}), 128.7,128.3,127.7\left(\mathrm{~d}, J_{\mathrm{CP}}=12.9 \mathrm{~Hz}\right), 127.5,127.3\left(\mathrm{~d}, J_{\mathrm{CP}}=1.0 \mathrm{~Hz}\right), 127.1$, $127.0,126.7,126.5\left(\mathrm{~d}, J_{\mathrm{CP}}=12.4 \mathrm{~Hz}\right), 125.1,123.5,119.1\left(\mathrm{~d}, J_{\mathrm{CP}}=5.1 \mathrm{~Hz}\right), 111.7,94.6(\mathrm{~d}$, $\left.J_{\mathrm{CP}}=8.3 \mathrm{~Hz}\right), 55.3 .{ }^{31} \mathrm{P}\left\{{ }^{1} \mathrm{H}\right\} \operatorname{NMR}\left(\mathrm{CDCl}_{3}\right): \delta 33.7(\mathrm{~s}) .[\alpha]^{20}{ }_{\mathrm{D}}+227\left(c 0.56, \mathrm{CHCl}_{3}\right)$. Anal. Calcd for $\mathrm{C}_{33} \mathrm{H}_{24} \mathrm{IO}_{2} \mathrm{P}: \mathrm{C}, 64.93 ; \mathrm{H}, 3.96$. Found: C, 64.92; H, 4.12. 


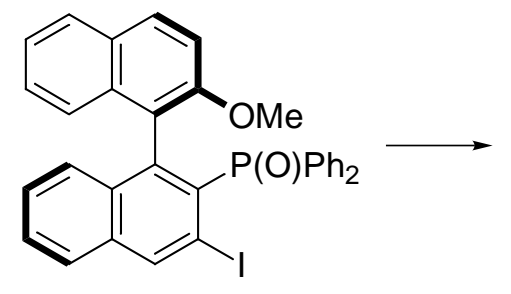

$(R)-5$

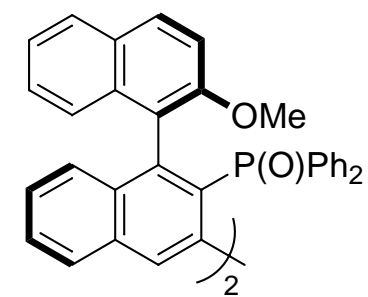

$(R, R)-\mathrm{S} 1$

A mixture of $(R)-5(545 \mathrm{mg}, 0.893 \mathrm{mmol})$ and copper powder $(210 \mathrm{mg}, 3.31 \mathrm{mmol})$ in DMF $(7.0 \mathrm{~mL})$ was refluxed for $40 \mathrm{~h}$. After removing the solvent under vacuum, the residue was diluted with $\mathrm{CHCl}_{3}$ and filtered through celite. The solvent was removed under vacuum and the residue was chromatographed on silica gel with EtOAc to afford $(R, R)$-S1 as a pale yellow solid (357 $\mathrm{mg}, 0.369 \mathrm{mmol} ; 83 \%$ yield).

${ }^{1} \mathrm{H} \mathrm{NMR}\left(\mathrm{CDCl}_{3}\right): \delta 7.61\left(\mathrm{dd},{ }^{2} \mathrm{~J}_{\mathrm{PH}}=11.8 \mathrm{~Hz}\right.$ and $\left.{ }^{3} \mathrm{~J}_{\mathrm{HH}}=7.2 \mathrm{~Hz}, 4 \mathrm{H}\right), 7.50\left(\mathrm{~d},{ }^{3} J_{\mathrm{HH}}=\right.$ $9.2 \mathrm{~Hz}, 2 \mathrm{H}), 7.41(\mathrm{t}, J=7.5 \mathrm{~Hz}, 4 \mathrm{H}), 7.36\left(\mathrm{~d},{ }^{4} J_{\mathrm{HH}}=3.2 \mathrm{~Hz}, 2 \mathrm{H}\right), 7.33-7.28(\mathrm{~m}, 4 \mathrm{H}), 7.22$ $\left(\mathrm{t},{ }^{3} \mathrm{~J}_{\mathrm{HH}}=7.7 \mathrm{~Hz}, 2 \mathrm{H}\right), 7.20-7.12(\mathrm{~m}, 12 \mathrm{H}), 7.06-7.02(\mathrm{~m}, 4 \mathrm{H}), 6.75-6.72(\mathrm{~m}, 4 \mathrm{H}), 6.55(\mathrm{td}$, ${ }^{3} J_{\mathrm{HH}}=7.8 \mathrm{~Hz}$ and $\left.{ }^{4} J_{\mathrm{HH}}=2.9 \mathrm{~Hz}, 4 \mathrm{H}\right), 3.79(\mathrm{~s}, 6 \mathrm{H}) .{ }^{13} \mathrm{C} \mathrm{NMR}\left(\mathrm{CDCl}_{3}\right): \delta 154.4,142.2$ $\left(\mathrm{dd}, J_{\mathrm{CP}}=8.6\right.$ and $\left.2.3 \mathrm{~Hz}\right), 139.6\left(\mathrm{~d}, J_{\mathrm{CP}}=9.8 \mathrm{~Hz}\right), 137.0\left(\mathrm{~d}, J_{\mathrm{CP}}=102.5 \mathrm{~Hz}\right), 135.2,134.8$ $\left(\mathrm{d}, J_{\mathrm{CP}}=104.0 \mathrm{~Hz}\right), 133.0\left(\mathrm{~d}, J_{\mathrm{CP}}=2.1 \mathrm{~Hz}\right), 132.6\left(\mathrm{~d}, J_{\mathrm{CP}}=9.4 \mathrm{~Hz}\right), 132.0\left(\mathrm{~d}, J_{\mathrm{CP}}=11.9\right.$ $\mathrm{Hz}), 131.49\left(\mathrm{~d}, J_{\mathrm{CP}}=102.5 \mathrm{~Hz}\right), 131.46\left(\mathrm{~d}, J_{\mathrm{CP}}=9.9 \mathrm{~Hz}\right), 131.3\left(\mathrm{~d}, J_{\mathrm{CP}}=10.4 \mathrm{~Hz}\right), 130.6$, $130.1\left(\mathrm{~d}, J_{\mathrm{CP}}=2.3 \mathrm{~Hz}\right), 128.9,128.3,127.9,127.53,127.50\left(\mathrm{~d}, J_{\mathrm{CP}}=11.9 \mathrm{~Hz}\right), 127.0$, $126.8,126.7,126.6,126.1,125.7\left(\mathrm{~d}, J_{\mathrm{CP}}=12.9 \mathrm{~Hz}\right), 123.3,120.9\left(\mathrm{~d}, J_{\mathrm{CP}}=5.1 \mathrm{~Hz}\right), 111.5$, 55.4. ${ }^{31} \mathrm{P}\left\{{ }^{1} \mathrm{H}\right\}$ NMR $\left(\mathrm{CDCl}_{3}\right): \delta 28.4(\mathrm{~s})$. $[\alpha]_{\mathrm{D}}^{20}+242$ (c 0.59, $\left.\mathrm{CHCl}_{3}\right)$. Anal. Calcd for $\mathrm{C}_{72} \mathrm{H}_{54} \mathrm{O}_{4} \mathrm{P}_{2}\left((R, R)-\mathrm{S} 1 \cdot \mathrm{C}_{6} \mathrm{H}_{6}\right): \mathrm{C}, 82.74 ; \mathrm{H}, 5.21$. Found: $\mathrm{C}, 87.70 ; \mathrm{H}, 5.22$.

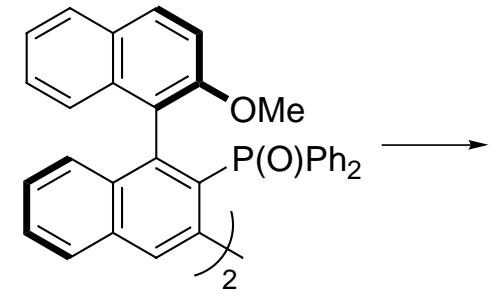

$(R, R)-\mathbf{S} 1$

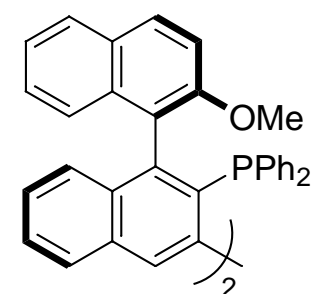

$(R, R)-3$

Methyl trifluoromethanesulfonate $(294 \mu \mathrm{L}, 2.60 \mathrm{mmol})$ was added to a solution of $(R, R)-S 1(418 \mathrm{mg}, 0.432 \mathrm{mmol})$ in DME $(26 \mathrm{~mL})$ at room temperature and the mixture was stirred for $3 \mathrm{~h}$ at $60{ }^{\circ} \mathrm{C}$. After cooling to $0{ }^{\circ} \mathrm{C}, \mathrm{LiAlH}_{4}(246 \mathrm{mg}, 6.48 \mathrm{mmol})$ was added to it and the resulting mixture was stirred for $30 \mathrm{~min}$ at $60{ }^{\circ} \mathrm{C}$. The reaction was then quenched by successive addition of water $(0.25 \mathrm{~mL}), \mathrm{NaOH}(0.25 \mathrm{~mL} ; 15 \%$ aqueous), and water $(0.75 \mathrm{~mL})$ at room temperature. This was diluted with $\mathrm{C}_{6} \mathrm{H}_{6}$ and filtered through celite. The resulting solution was dried over $\mathrm{MgSO}_{4}$, filtered, 
and concentrated under vacuum. The residue was chromatographed on silica gel with $\mathrm{C}_{6} \mathrm{H}_{6}$ to afford $(R, R)-3$ as a pale yellow solid (360 mg, $0.385 \mathrm{mmol} ; 89 \%$ yield).

${ }^{1} \mathrm{H}$ NMR $\left(\mathrm{CDCl}_{3}\right): \delta$ 7.44-7.36 (m, 6H), 7.31 (bs, 4H), 7.28-7.18 (m, 6H), 7.12-7.06 $(\mathrm{m}, 8 \mathrm{H}), 7.03\left(\mathrm{t},{ }^{3} J_{\mathrm{HH}}=7.6 \mathrm{~Hz}, 4 \mathrm{H}\right), 6.97\left(\mathrm{~d},{ }^{3} J_{\mathrm{HH}}=9.1 \mathrm{~Hz}, 2 \mathrm{H}\right), 6.76\left(\mathrm{~d},{ }^{3} J_{\mathrm{HH}}=8.6 \mathrm{~Hz}\right.$, $2 \mathrm{H}), 6.72(\mathrm{bs}, 4 \mathrm{H}), 6.54\left(\mathrm{t},{ }^{3} J_{\mathrm{HH}}=7.3 \mathrm{~Hz}, 2 \mathrm{H}\right), 6.36\left(\mathrm{t},{ }^{3} J_{\mathrm{HH}}=7.5 \mathrm{~Hz}, 4 \mathrm{H}\right), 3.69(\mathrm{~s}, 6 \mathrm{H})$. ${ }^{13} \mathrm{C} \mathrm{NMR}\left(\mathrm{CDCl}_{3}\right): \delta 154.5,143.4\left(\mathrm{t}, J_{\mathrm{CP}}=19.3 \mathrm{~Hz}\right), 139.0\left(\mathrm{t}, J_{\mathrm{CP}}=1.6 \mathrm{~Hz}\right), 138.1\left(\mathrm{t}, J_{\mathrm{CP}}=\right.$ $2.6 \mathrm{~Hz}), 135.8\left(\mathrm{t}, J_{\mathrm{CP}}=9.4 \mathrm{~Hz}\right), 135.0,134.8\left(\mathrm{t}, J_{\mathrm{CP}}=11.1 \mathrm{~Hz}\right), 134.5\left(\mathrm{~d}, J_{\mathrm{CP}}=8.8 \mathrm{~Hz}\right)$, $133.6,133.3\left(\mathrm{t}, J_{\mathrm{CP}}=12.1 \mathrm{~Hz}\right), 133.2,131.4\left(\mathrm{t}, J_{\mathrm{CP}}=4.9 \mathrm{~Hz}\right), 129.7,128.6,128.3,127.7$, $127.6,127.4\left(\mathrm{t}, J_{\mathrm{CP}}=3.8 \mathrm{~Hz}\right), 127.2,126.6,126.4\left(\mathrm{t}, J_{\mathrm{CP}}=3.4 \mathrm{~Hz}\right), 126.1,125.7,125.6$, 125.5, 122.9, 120.8, 111.8, 55.3. ${ }^{31} \mathrm{P}\left\{{ }^{1} \mathrm{H}\right\} \operatorname{NMR}\left(\mathrm{CDCl}_{3}\right): \delta 1.8(\mathrm{~s}) .[\alpha]_{\mathrm{D}}^{20}+488(c 0.58$, $\mathrm{CHCl}_{3}$ ). Anal. Calcd for $\mathrm{C}_{66} \mathrm{H}_{48} \mathrm{O}_{2} \mathrm{P}_{2}: \mathrm{C}, 84.78 ; \mathrm{H}$, 5.17. Found: $\mathrm{C}, 84.60 ; \mathrm{H}, 5.45$.

\section{Catalytic Reactions}

\section{General Procedure for Table 1.}

A solution of $\left[\mathrm{Rh}(\mathrm{cod})_{2}\right] \mathrm{BF}_{4}(4.1 \mathrm{mg}, 10 \mu \mathrm{mol} \mathrm{Rh})$ and ligand $(11.0 \mu \mathrm{mol})$ in THF $(2.5 \mathrm{~mL})$ was stirred for $20 \mathrm{~min}$ at $60{ }^{\circ} \mathrm{C}$. Propargyl alcohol $1(0.20 \mathrm{mmol})$ and $\mathrm{KOH}$ (50 $\mu \mathrm{L}, 30 \mu \mathrm{mol} ; 0.6 \mathrm{M}$ aqueous) were then added to it. After stirring for $24 \mathrm{~h}$ at 60 ${ }^{\circ} \mathrm{C}$, the reaction mixture was directly passed through a pad of silica gel with $\mathrm{Et}_{2} \mathrm{O}$ and the solvent was removed under vacuum. The residue was purified by silica gel preparative TLC with $\mathrm{Et}_{2} \mathrm{O} /$ hexane to afford indanone 2.

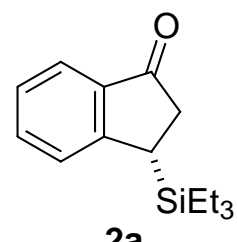

Entry 5. (CAS 849518-58-7) Pale yellow oil. 57\% yield. The ee was determined on a Daicel Chiralcel OD-H column with hexane : 2-propanol $=95: 5$, flow $=1.0 \mathrm{~mL} / \mathrm{min}$. Retention times: $4.8 \mathrm{~min}\left[(R)\right.$-enantiomer], $6.1 \mathrm{~min}\left[(S)\right.$-enantiomer]. $74 \%$ ee. $[\alpha]^{20}{ }_{\mathrm{D}}$ $-117\left(c 0.74, \mathrm{CHCl}_{3}\right)$. The absolute configuration was assigned by analogy with entry 8.

${ }^{1} \mathrm{H}$ NMR $\left(\mathrm{CDCl}_{3}\right): \delta 7.73\left(\mathrm{~d},{ }^{3} J_{\mathrm{HH}}=7.7 \mathrm{~Hz}, 1 \mathrm{H}\right), 7.53\left(\mathrm{t},{ }^{3} \mathrm{~J}_{\mathrm{HH}}=7.2 \mathrm{~Hz}, 1 \mathrm{H}\right), 7.36(\mathrm{~d}$, $\left.{ }^{3} J_{\mathrm{HH}}=7.8 \mathrm{~Hz}, 1 \mathrm{H}\right), 7.27\left(\mathrm{t},{ }^{3} J_{\mathrm{HH}}=7.5 \mathrm{~Hz}, 1 \mathrm{H}\right), 2.91\left(\mathrm{~d},{ }^{3} \mathrm{~J}_{\mathrm{HH}}=10.0 \mathrm{~Hz}, 1 \mathrm{H}\right), 2.87(\mathrm{dd}$, 
${ }^{2} J_{\mathrm{HH}}=18.1 \mathrm{~Hz}$ and $\left.{ }^{3} J_{\mathrm{HH}}=8.5 \mathrm{~Hz}, 1 \mathrm{H}\right), 2.62\left(\mathrm{~d},{ }^{2} J_{\mathrm{HH}}=18.2 \mathrm{~Hz}, 1 \mathrm{H}\right), 0.89\left(\mathrm{t},{ }^{3} J_{\mathrm{HH}}=7.8\right.$ $\mathrm{Hz}, 9 \mathrm{H}), 0.58\left(\mathrm{q},{ }^{3} J_{\mathrm{HH}}=8.0 \mathrm{~Hz}, 6 \mathrm{H}\right) .{ }^{13} \mathrm{C} \mathrm{NMR}\left(\mathrm{CDCl}_{3}\right): \delta 207.2,159.0,136.2,134.3$, $125.73,125.68,124.0,39.4,25.3,7.3,2.3$.

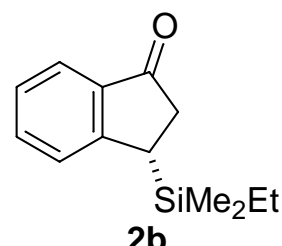

Entry 8. Pale yellow oil. 57\% yield. The ee was determined on a Daicel Chiralpak AD-H column with hexane : 2-propanol $=100: 1$, flow $=0.5 \mathrm{~mL} / \mathrm{min}$. Retention times: $17.7 \min \left[(R)\right.$-enantiomer], $18.4 \mathrm{~min}\left[(S)\right.$-enantiomer]. $99 \%$ ee. $[\alpha]^{20}{ }_{D}-155(c$ $\left.0.96, \mathrm{CHCl}_{3}\right)$. The absolute configuration was determined to be $(S)$ by converting it to 1-indanol (see below).

${ }^{1} \mathrm{H} \mathrm{NMR}\left(\mathrm{CDCl}_{3}\right): \delta 7.74\left(\mathrm{~d},{ }^{3} J_{\mathrm{HH}}=7.5 \mathrm{~Hz}, 1 \mathrm{H}\right), 7.54\left(\mathrm{t},{ }^{3} J_{\mathrm{HH}}=7.5 \mathrm{~Hz}, 1 \mathrm{H}\right), 7.37(\mathrm{~d}$, $\left.{ }^{3} J_{\mathrm{HH}}=7.8 \mathrm{~Hz}, 1 \mathrm{H}\right), 7.28\left(\mathrm{t},{ }^{3} J_{\mathrm{HH}}=7.7 \mathrm{~Hz}, 1 \mathrm{H}\right), 2.87\left(\mathrm{dd},{ }^{2} J_{\mathrm{HH}}=18.9 \mathrm{~Hz}\right.$ and ${ }^{3} J_{\mathrm{HH}}=8.6$ $\mathrm{Hz}, 1 \mathrm{H}), 2.81\left(\mathrm{~d},{ }^{3} J_{\mathrm{HH}}=8.8 \mathrm{~Hz}, 1 \mathrm{H}\right), 2.58\left(\mathrm{dd},{ }^{2} J_{\mathrm{HH}}=18.9 \mathrm{~Hz}\right.$ and $\left.{ }^{3} J_{\mathrm{HH}}=1.6 \mathrm{~Hz}, 1 \mathrm{H}\right)$, $0.91\left(\mathrm{t},{ }^{3} J_{\mathrm{HH}}=7.9 \mathrm{~Hz}, 3 \mathrm{H}\right), 0.54\left(\mathrm{q},{ }^{3} J_{\mathrm{HH}}=7.9 \mathrm{~Hz}, 2 \mathrm{H}\right),-0.01(\mathrm{~s}, 3 \mathrm{H}),-0.04(\mathrm{~s}, 3 \mathrm{H}) .{ }^{13} \mathrm{C}$ NMR $\left(\mathrm{CDCl}_{3}\right): \delta 207.2,158.8,136.2,134.4,125.8,125.7,124.0,39.6,27.6,7.2,5.5,-5.1$, -5.5. Anal. Calcd for $\mathrm{C}_{13} \mathrm{H}_{18} \mathrm{OSi}$ : C, 71.50; $\mathrm{H}, 8.31$. Found: $\mathrm{C}, 71.77 ; \mathrm{H}, 8.52$.<smiles>CCN(CC)[C@H]1CC(=O)c2ccccc21</smiles>

$2 b$

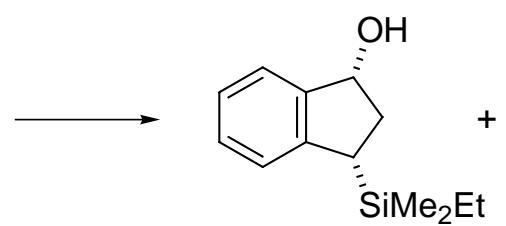

cis-S2

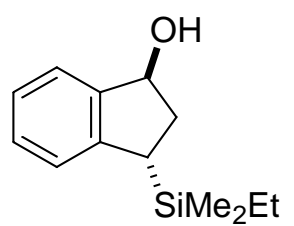

trans-S2

$\mathrm{LiAlH}_{4}(18 \mathrm{mg}, 0.47 \mathrm{mmol})$ was added to a solution of $\mathbf{2 b}(102 \mathrm{mg}, 0.47 \mathrm{mmol})$ in $\mathrm{Et}_{2} \mathrm{O}(3.0 \mathrm{~mL})$ at $0{ }^{\circ} \mathrm{C}$ and the mixture was stirred for $30 \mathrm{~min}$ at $0{ }^{\circ} \mathrm{C}$. The reaction was quenched with water, and this was passed through a pad of silica gel with $\mathrm{Et}_{2} \mathrm{O}$. After removing the solvent under vacuum, the residue was purified by silica gel preparative TLC with $\mathrm{Et}_{2} \mathrm{O} /$ hexane $=1 / 3$ to afford cis-S2 as a pale yellow oil ( $45 \mathrm{mg}$, $0.20 \mathrm{mmol}$; 43\% yield) and trans-S2 as a pale yellow oil (14 mg, $64 \mu \mathrm{mol} ; 13 \%$ yield).

cis-S2: $[\alpha]^{20}{ }_{\mathrm{D}}+42.4\left(c 1.64, \mathrm{CHCl}_{3}\right) .{ }^{1} \mathrm{H} \mathrm{NMR}\left(\mathrm{CDCl}_{3}\right): \delta 7.37\left(\mathrm{~d},{ }^{3} J_{\mathrm{HH}}=7.0 \mathrm{~Hz}, 1 \mathrm{H}\right)$, 7.23-7.20 (m, 1H), 7.18-7.15 $(\mathrm{m}, 2 \mathrm{H}), 5.28(\mathrm{~m}, 1 \mathrm{H}), 2.62\left(\mathrm{ddd},{ }^{2} J_{\mathrm{HH}}=13.4 \mathrm{~Hz}\right.$ and ${ }^{3} J_{\mathrm{HH}}=$ 9.2 and $7.5 \mathrm{~Hz}, 1 \mathrm{H}), 2.52\left(\mathrm{dd},{ }^{3} J_{\mathrm{HH}}=8.9\right.$ and $\left.6.6 \mathrm{~Hz}, 1 \mathrm{H}\right), 1.84\left(\mathrm{ddd},{ }^{2} J_{\mathrm{HH}}=13.4 \mathrm{~Hz}\right.$ and ${ }^{3} J_{\mathrm{HH}}=6.5$ and $\left.4.9 \mathrm{~Hz}, 1 \mathrm{H}\right), 1.64\left(\mathrm{~d},{ }^{3} J_{\mathrm{HH}}=5.8 \mathrm{~Hz}, 1 \mathrm{H}\right), 0.96\left(\mathrm{t},{ }^{3} J_{\mathrm{HH}}=7.8 \mathrm{~Hz}, 3 \mathrm{H}\right)$ 
0.65-0.59 (m, 2H), $0.05(\mathrm{~s}, 3 \mathrm{H}), 0.03(\mathrm{~s}, 3 \mathrm{H}) .{ }^{13} \mathrm{C} \mathrm{NMR}\left(\mathrm{CDCl}_{3}\right): \delta 146.0,144.8,128.3$, 125.6, 124.3, 124.1, 76.6, 37.6, 30.7, 7.3, 6.1, -4.6, -4.9. HRMS (ESI) calcd for $\mathrm{C}_{13} \mathrm{H}_{20} \mathrm{OSiNa}\left(\mathrm{M}+\mathrm{Na}^{+}\right)$243.1176, found 243.1172.

trans-S2: $[\alpha]^{20}+44.1\left(c 0.65, \mathrm{CHCl}_{3}\right) .{ }^{1} \mathrm{H}$ NMR $\left(\mathrm{CDCl}_{3}\right): \delta 7.38\left(\mathrm{~d},{ }^{3} \mathrm{~J}_{\mathrm{HH}}=7.3 \mathrm{~Hz}\right.$, $1 \mathrm{H}), 7.21\left(\mathrm{t},{ }^{3} \mathrm{~J}_{\mathrm{HH}}=7.3 \mathrm{~Hz}, 1 \mathrm{H}\right), 7.16\left(\mathrm{t},{ }^{3} \mathrm{~J}_{\mathrm{HH}}=7.3 \mathrm{~Hz}, 1 \mathrm{H}\right), 7.11\left(\mathrm{~d},{ }^{3} J_{\mathrm{HH}}=7.5 \mathrm{~Hz}, 1 \mathrm{H}\right)$, $5.21(\mathrm{bs}, 1 \mathrm{H}), 2.66\left(\mathrm{dd},{ }^{3} J_{\mathrm{HH}}=9.8\right.$ and $\left.3.4 \mathrm{~Hz}, 1 \mathrm{H}\right), 2.49\left(\mathrm{ddd},{ }^{2} J_{\mathrm{HH}}=13.1 \mathrm{~Hz}\right.$ and ${ }^{3} J_{\mathrm{HH}}=$ 7.2 and $3.3 \mathrm{~Hz}, 1 \mathrm{H}), 2.13\left(\mathrm{ddd},{ }^{2} J_{\mathrm{HH}}=13.0 \mathrm{~Hz}\right.$ and ${ }^{3} J_{\mathrm{HH}}=9.5$ and $\left.6.9 \mathrm{~Hz}, 1 \mathrm{H}\right), 1.68(\mathrm{bs}$, $1 \mathrm{H}), 0.92\left(\mathrm{t},{ }^{3} \mathrm{JHH}_{\mathrm{HH}}=7.9 \mathrm{~Hz}, 3 \mathrm{H}\right), 0.54\left(\mathrm{q},{ }^{3} \mathrm{~J}_{\mathrm{HH}}=8.0 \mathrm{~Hz}, 2 \mathrm{H}\right),-0.04(\mathrm{~s}, 3 \mathrm{H}),-0.05(\mathrm{~s}, 3 \mathrm{H})$. ${ }^{13} \mathrm{C}$ NMR $\left(\mathrm{CDCl}_{3}\right): \delta 145.6,144.1,128.1,125.4,124.1,124.0,76.3,38.8,30.7,7.3,5.8$, $-4.9,-5.1$. HRMS (ESI) calcd for $\mathrm{C}_{13} \mathrm{H}_{20} \mathrm{OSiNa}\left(\mathrm{M}+\mathrm{Na}^{+}\right)$243.1176, found 243.1173.

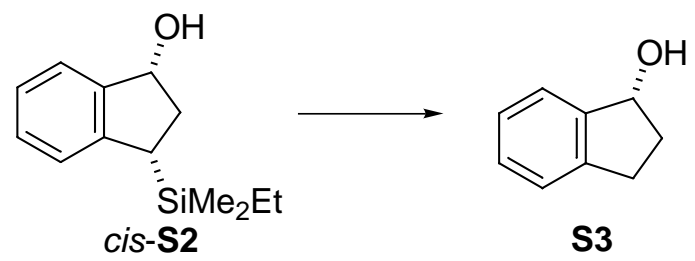

TBAF (1.0 mL, $1.0 \mathrm{mmol} ; 1.0 \mathrm{M}$ solution in THF) was added to a solution of $c$ is-S2 $(45 \mathrm{mg}, 0.20 \mathrm{mmol})$ in THF $(1.0 \mathrm{~mL})$ at $0{ }^{\circ} \mathrm{C}$ and the mixture was stirred for $3 \mathrm{~h}$ at room temperature. The reaction was quenched with water and extracted with $\mathrm{Et}_{2} \mathrm{O}$. The organic layer was dried over $\mathrm{MgSO}_{4}$, filtered, and concentrated under vacuum. The residue was purified by silica gel preparative TLC with $\mathrm{Et}_{2} \mathrm{O}$ / hexane $=1 / 2$ to afford 1-indanol S3 (CAS 6351-10-6, 697-64-3 for $(R)$ ) as a white solid (21 mg, 0.15 mmol; $76 \%$ yield $) .[\alpha]^{20}{ }_{D}-26.7\left(c 1.03, \mathrm{CHCl}_{3}\right)$.

${ }^{1} \mathrm{H}$ NMR $\left(\mathrm{CDCl}_{3}\right): \delta 7.42\left(\mathrm{~d},{ }^{3} \mathrm{~J}_{\mathrm{HH}}=6.0 \mathrm{~Hz}, 1 \mathrm{H}\right), 7.29-7.22(\mathrm{~m}, 3 \mathrm{H}), 5.25\left(\mathrm{t},{ }^{3} \mathrm{~J}_{\mathrm{HH}}=6.0\right.$ $\mathrm{Hz}, 1 \mathrm{H}), 3.06\left(\mathrm{ddd},{ }^{2} J_{\mathrm{HH}}=16.0 \mathrm{~Hz}\right.$ and ${ }^{3} J_{\mathrm{HH}}=8.5$ and $\left.4.7 \mathrm{~Hz}, 1 \mathrm{H}\right), 2.82\left(\mathrm{ddd},{ }^{2} J_{\mathrm{HH}}=\right.$ $15.6 \mathrm{~Hz}$ and ${ }^{3} \mathrm{~J}_{\mathrm{HH}}=8.0$ and $\left.6.8 \mathrm{~Hz}, 1 \mathrm{H}\right), 2.49\left(\mathrm{dddd},{ }^{2} J_{\mathrm{HH}}=13.2 \mathrm{~Hz}\right.$ and ${ }^{3} \mathrm{~J}_{\mathrm{HH}}=8.3,6.8$, and $4.9 \mathrm{~Hz}, 1 \mathrm{H}), 1.95\left(\mathrm{dddd},{ }^{2} J_{\mathrm{HH}}=13.6 \mathrm{~Hz}\right.$ and ${ }^{3} J_{\mathrm{HH}}=8.5,6.6$, and $\left.5.2 \mathrm{~Hz}, 1 \mathrm{H}\right), 1.73$ (bs, 1H).

The absolute configuration of $\mathbf{S} 3$ was determined to be $(R)$ by comparison of the optical rotation with the literature value $\left([\alpha]^{20}{ }_{\mathrm{D}}+34.4\left(c 1.97, \mathrm{CHCl}_{3}\right)\right.$ for $\left.(S)\right){ }^{5}$ This assignment leads to the determination of the absolute configuration of indanone $\mathbf{2 b}$ as $(S)$.

\footnotetext{
${ }^{5}$ Hückel, W.; Mossner, F. Ann. 1960, 637, 57.
} 


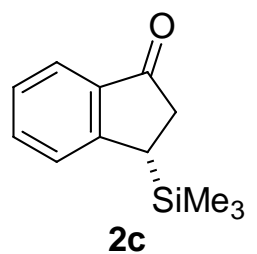

Entry 9. (CAS 849518-57-6) Pale yellow oil. 44\% yield. The ee was determined on a Daicel Chiralpak AD-H column with hexane : 2-propanol $=98: 2$, flow $=0.5$ $\mathrm{mL} / \mathrm{min}$. Retention times: $18.1 \mathrm{~min}$ [(R)-enantiomer], $20.2 \mathrm{~min}$ [(S)-enantiomer]. $92 \%$ ee. $[\alpha]_{\mathrm{D}}^{20}-155\left(c 0.64, \mathrm{CHCl}_{3}\right)$. The absolute configuration was assigned by analogy with entry 8 .

${ }^{1} \mathrm{H}$ NMR $\left(\mathrm{CDCl}_{3}\right): \delta 7.74\left(\mathrm{~d},{ }^{3} J_{\mathrm{HH}}=7.7 \mathrm{~Hz}, 1 \mathrm{H}\right), 7.54\left(\mathrm{t},{ }^{3} J_{\mathrm{HH}}=7.6 \mathrm{~Hz}, 1 \mathrm{H}\right), 7.37(\mathrm{~d}$, $\left.{ }^{3} J_{\mathrm{HH}}=7.8 \mathrm{~Hz}, 1 \mathrm{H}\right), 7.28\left(\mathrm{t},{ }^{3} J_{\mathrm{HH}}=7.7 \mathrm{~Hz}, 1 \mathrm{H}\right), 2.87\left(\mathrm{dd},{ }^{2} J_{\mathrm{HH}}=19.4 \mathrm{~Hz}\right.$ and ${ }^{3} J_{\mathrm{HH}}=8.5$ $\mathrm{Hz}, 1 \mathrm{H}), 2.79-2.76(\mathrm{~m}, 1 \mathrm{H}), 2.57\left(\mathrm{dd},{ }^{2} J_{\mathrm{HH}}=19.3 \mathrm{~Hz}\right.$ and $\left.{ }^{3} J_{\mathrm{HH}}=2.0 \mathrm{~Hz}, 1 \mathrm{H}\right), 0.02(\mathrm{~s}$, $9 \mathrm{H}) .{ }^{13} \mathrm{C} \mathrm{NMR}\left(\mathrm{CDCl}_{3}\right): \delta 207.1,158.6,136.0,134.3,125.8,125.6,123.9,39.5,28.7,-3.2$.

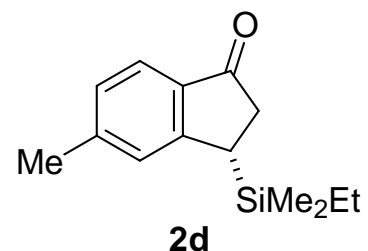

Entry 10. Pale yellow oil. $60 \%$ yield. The ee was determined on a Daicel Chiralpak AD-H column with hexane : 2-propanol $=100: 1$, flow $=0.5 \mathrm{~mL} / \mathrm{min}$. Retention times: $23.8 \mathrm{~min}\left[(R)\right.$-enantiomer], $29.0 \mathrm{~min}\left[(S)\right.$-enantiomer]. 93\% ee. $[\alpha]^{20}{ }_{\mathrm{D}}-133(c$ $\left.0.93, \mathrm{CHCl}_{3}\right)$. The absolute configuration was assigned by analogy with entry 8 .

${ }^{1} \mathrm{H} \mathrm{NMR}\left(\mathrm{CDCl}_{3}\right): \delta 7.61\left(\mathrm{~d},{ }^{3} J_{\mathrm{HH}}=7.8 \mathrm{~Hz}, 1 \mathrm{H}\right), 7.13(\mathrm{~s}, 1 \mathrm{H}), 7.08\left(\mathrm{~d},{ }^{3} J_{\mathrm{HH}}=7.8 \mathrm{~Hz}\right.$, $1 \mathrm{H}), 2.84\left(\mathrm{dd},{ }^{2} J_{\mathrm{HH}}=19.1 \mathrm{~Hz}\right.$ and $\left.{ }^{3} J_{\mathrm{HH}}=8.4 \mathrm{~Hz}, 1 \mathrm{H}\right), 2.73\left(\mathrm{~d},{ }^{3} J_{\mathrm{HH}}=9.2 \mathrm{~Hz}, 1 \mathrm{H}\right), 2.54$ $\left(\mathrm{dd},{ }^{2} J_{\mathrm{HH}}=19.3 \mathrm{~Hz}\right.$ and $\left.{ }^{3} \mathrm{~J}_{\mathrm{HH}}=2.1 \mathrm{~Hz}, 1 \mathrm{H}\right), 2.41(\mathrm{~s}, 3 \mathrm{H}), 0.90\left(\mathrm{t}^{3}{ }^{3} \mathrm{JHH}_{\mathrm{HH}}=7.9 \mathrm{~Hz}, 3 \mathrm{H}\right), 0.53$ $\left(\mathrm{q},{ }^{3} J_{\mathrm{HH}}=7.8 \mathrm{~Hz}, 2 \mathrm{H}\right),-0.02(\mathrm{~s}, 3 \mathrm{H}),-0.05(\mathrm{~s}, 3 \mathrm{H}) .{ }^{13} \mathrm{C} \mathrm{NMR}\left(\mathrm{CDCl}_{3}\right): \delta 206.6,159.3$, 145.5, 133.9, 127.2, 126.0, 123.8, 39.7, 27.3, 22.2, 7.2, 5.5, -5.1, -5.5. Anal. Calcd for $\mathrm{C}_{14} \mathrm{H}_{20} \mathrm{OSi}: \mathrm{C}, 72.36 ; \mathrm{H}, 8.67$. Found: $\mathrm{C}, 72.21 ; \mathrm{H}, 8.64$. 


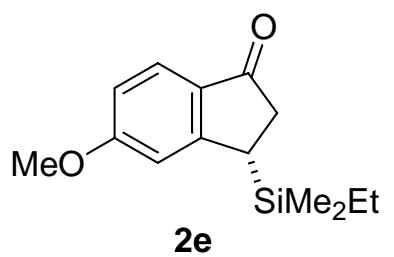

Entry 11. Pale yellow oil. 57\% yield. The ee was determined on a Daicel Chiralpak AD-H column with hexane $:$ 2-propanol $=100: 1$, flow $=1.0 \mathrm{~mL} / \mathrm{min}$. Retention times: $25.9 \min \left[(R)\right.$-enantiomer], $34.5 \mathrm{~min}\left[(S)\right.$-enantiomer]. $94 \%$ ee. $[\alpha]^{20}{ }_{\mathrm{D}}-87.8(c$ 1.31, $\mathrm{CHCl}_{3}$ ). The absolute configuration was assigned by analogy with entry 8 .

${ }^{1} \mathrm{H} \mathrm{NMR}\left(\mathrm{CDCl}_{3}\right): \delta 7.66\left(\mathrm{~d},{ }^{3} J_{\mathrm{HH}}=8.5 \mathrm{~Hz}, 1 \mathrm{H}\right), 6.81\left(\mathrm{dd},{ }^{3} J_{\mathrm{HH}}=8.5 \mathrm{~Hz}\right.$ and ${ }^{4} J_{\mathrm{HH}}=$ $2.2 \mathrm{~Hz}, 1 \mathrm{H}), 6.77\left(\mathrm{~d},{ }^{4} J_{\mathrm{HH}}=1.8 \mathrm{~Hz}, 1 \mathrm{H}\right), 3.86(\mathrm{~s}, 3 \mathrm{H}), 2.84\left(\mathrm{dd},{ }^{2} J_{\mathrm{HH}}=19.1 \mathrm{~Hz}\right.$ and ${ }^{3} J_{\mathrm{HH}}$ $=8.5 \mathrm{~Hz}, 1 \mathrm{H}), 2.74\left(\mathrm{~d},{ }^{3} J_{\mathrm{HH}}=8.5 \mathrm{~Hz}, 1 \mathrm{H}\right), 2.54\left(\mathrm{dd},{ }^{2} J_{\mathrm{HH}}=19.2 \mathrm{~Hz}\right.$ and ${ }^{3} J_{\mathrm{HH}}=2.0 \mathrm{~Hz}$, $1 \mathrm{H}), 0.91\left(\mathrm{t},{ }^{3} \mathrm{~J}_{\mathrm{HH}}=7.9 \mathrm{~Hz}, 3 \mathrm{H}\right), 0.54\left(\mathrm{q},{ }^{3} \mathrm{~J}_{\mathrm{HH}}=7.8 \mathrm{~Hz}, 2 \mathrm{H}\right),-0.01(\mathrm{~s}, 3 \mathrm{H}),-0.04(\mathrm{~s}, 3 \mathrm{H})$. ${ }^{13} \mathrm{C}$ NMR $\left(\mathrm{CDCl}_{3}\right): \delta 205.3,165.0,161.6,129.6,125.6,113.5,109.1,55.5,39.7,27.6,7.2$, 5.5, -5.1, -5.5. Anal. Calcd for $\mathrm{C}_{14} \mathrm{H}_{20} \mathrm{O}_{2} \mathrm{Si}$ : C, 67.70; H, 8.12. Found: $\mathrm{C}, 67.89 ; \mathrm{H}, 8.34$.

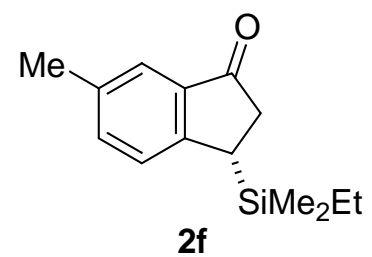

Entry 12. Pale yellow oil. 56\% yield ( $>20 / 1$ regioselectivity). The ee was determined on a Daicel Chiralpak AD-H column with hexane : 2-propanol $=100: 1$, flow $=0.5 \mathrm{~mL} / \mathrm{min}$. Retention times: $21.1 \mathrm{~min}[(S)$-enantiomer], $24.0 \mathrm{~min}[(R)-$ enantiomer]. $92 \%$ ee. $[\alpha]^{20}-129\left(c 0.92, \mathrm{CHCl}_{3}\right)$. The absolute configuration was assigned by analogy with entry 8 .

${ }^{1} \mathrm{H} \mathrm{NMR}\left(\mathrm{CDCl}_{3}\right): \delta 7.53(\mathrm{~s}, 1 \mathrm{H}), 7.35\left(\mathrm{dd},{ }^{3} J_{\mathrm{HH}}=7.9 \mathrm{~Hz}\right.$ and $\left.{ }^{4} J_{\mathrm{HH}}=1.6 \mathrm{~Hz}, 1 \mathrm{H}\right)$, $7.24\left(\mathrm{~d},{ }^{3} J_{\mathrm{HH}}=7.9 \mathrm{~Hz}, 1 \mathrm{H}\right), 2.85\left(\mathrm{dd},{ }^{2} J_{\mathrm{HH}}=19.3 \mathrm{~Hz}\right.$ and $\left.{ }^{3} J_{\mathrm{HH}}=8.4 \mathrm{~Hz}, 1 \mathrm{H}\right), 2.75(\mathrm{~d}$, $\left.{ }^{3} J_{\mathrm{HH}}=8.5 \mathrm{~Hz}, 1 \mathrm{H}\right), 2.56\left(\mathrm{dd},{ }^{2} J_{\mathrm{HH}}=19.3 \mathrm{~Hz}\right.$ and $\left.{ }^{3} J_{\mathrm{HH}}=1.9 \mathrm{~Hz}, 1 \mathrm{H}\right), 2.38(\mathrm{~s}, 3 \mathrm{H}), 0.91(\mathrm{t}$, $\left.{ }^{3} \mathrm{~J}_{\mathrm{HH}}=7.9 \mathrm{~Hz}, 3 \mathrm{H}\right), 0.53\left(\mathrm{q},{ }^{3} \mathrm{~J}_{\mathrm{HH}}=7.9 \mathrm{~Hz}, 2 \mathrm{H}\right),-0.02(\mathrm{~s}, 3 \mathrm{H}),-0.05(\mathrm{~s}, 3 \mathrm{H}) .{ }^{13} \mathrm{C} \mathrm{NMR}$ $\left(\mathrm{CDCl}_{3}\right): \delta 207.3,156.1,136.3,135.7,135.6,125.3,123.9,39.8,27.0,20.9,7.2,5.5,-5.1$, -5.5. Anal. Calcd for $\mathrm{C}_{14} \mathrm{H}_{20} \mathrm{OSi}$ : C, 72.36; H, 8.67. Found: $\mathrm{C}, 72.30 ; \mathrm{H}, 8.61$. 


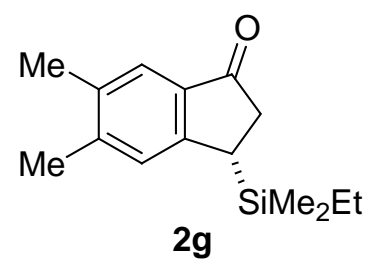

Entry 13. Pale yellow oil. 50\% yield ( $>20 / 1$ regioselectivity). The ee was determined on a Daicel Chiralcel OD-H column with hexane : 2-propanol = $98: 2$, flow $=1.0 \mathrm{~mL} / \mathrm{min}$. Retention times: $6.9 \mathrm{~min}[(R)$-enantiomer], $8.5 \mathrm{~min}[(S)$ enantiomer]. $95 \%$ ee. $[\alpha]^{20}-132\left(c 0.97, \mathrm{CHCl}_{3}\right)$. The absolute configuration was assigned by analogy with entry 8 .

${ }^{1} \mathrm{H}$ NMR $\left(\mathrm{CDCl}_{3}\right): \delta 7.49(\mathrm{~s}, 1 \mathrm{H}), 7.11(\mathrm{~s}, 1 \mathrm{H}), 2.82\left(\mathrm{dd},{ }^{2} \mathrm{~J}_{\mathrm{HH}}=19.3 \mathrm{~Hz}\right.$ and ${ }^{3} \mathrm{~J}_{\mathrm{HH}}=$ $8.4 \mathrm{~Hz}, 1 \mathrm{H}), 2.71\left(\mathrm{~d},{ }^{3} J_{\mathrm{HH}}=8.7 \mathrm{~Hz}, 1 \mathrm{H}\right), 2.53\left(\mathrm{dd},{ }^{2} J_{\mathrm{HH}}=19.3 \mathrm{~Hz}\right.$ and ${ }^{3} J_{\mathrm{HH}}=2.1 \mathrm{~Hz}$, $1 \mathrm{H}), 2.33(\mathrm{~s}, 3 \mathrm{H}), 2.28(\mathrm{~s}, 3 \mathrm{H}), 0.91\left(\mathrm{t},{ }^{3} J_{\mathrm{HH}}=7.9 \mathrm{~Hz}, 3 \mathrm{H}\right), 0.53\left(\mathrm{q},{ }^{3} \mathrm{~J}_{\mathrm{HH}}=7.8 \mathrm{~Hz}, 2 \mathrm{H}\right)$, $-0.02(\mathrm{~s}, 3 \mathrm{H}),-0.05(\mathrm{~s}, 3 \mathrm{H}) .{ }^{13} \mathrm{C} \mathrm{NMR}\left(\mathrm{CDCl}_{3}\right): \delta 206.9,156.9,144.5,134.7,134.4,126.4$, $124.3,39.7,26.9,20.9,19.6,7.2,5.6,-5.1,-5.5$. HRMS (ESI) calcd for $\mathrm{C}_{15} \mathrm{H}_{23} \mathrm{OSi}$ $\left(\mathrm{M}+\mathrm{H}^{+}\right)$247.1513, found 247.1515.

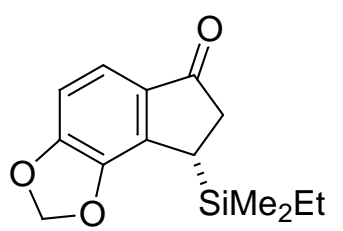

2h

Entry 14. Pale yellow oil. 55\% yield (10/1 regioselectivity). The ee was determined on a Daicel Chiralpak AS column with hexane : 2-propanol $=98: 2$, flow $=1.0$ $\mathrm{mL} / \mathrm{min}$. Retention times: $39.2 \mathrm{~min}$ [(S)-enantiomer], $62.6 \mathrm{~min}$ [(R)-enantiomer]. $96 \%$ ee. $[\alpha]_{\mathrm{D}}^{20}-5.9\left(\mathrm{c} 0.96, \mathrm{CHCl}_{3}\right)$. The absolute configuration was assigned by analogy with entry 8 .

${ }^{1} \mathrm{H} \mathrm{NMR}\left(\mathrm{CDCl}_{3}\right): \delta 7.37\left(\mathrm{~d},{ }^{3} J_{\mathrm{HH}}=8.1 \mathrm{~Hz}, 1 \mathrm{H}\right), 6.80\left(\mathrm{~d},{ }^{3} J_{\mathrm{HH}}=7.9 \mathrm{~Hz}, 1 \mathrm{H}\right), 6.05(\mathrm{~d}$, $\left.{ }^{2} J_{\mathrm{HH}}=18.3 \mathrm{~Hz}, 1 \mathrm{H}\right), 6.04\left(\mathrm{~d},{ }^{2} J_{\mathrm{HH}}=18.3 \mathrm{~Hz}, 1 \mathrm{H}\right), 2.86\left(\mathrm{dd},{ }^{2} J_{\mathrm{HH}}=18.8 \mathrm{~Hz}\right.$ and ${ }^{3} J_{\mathrm{HH}}=$ $8.7 \mathrm{~Hz}, 1 \mathrm{H}), 2.79\left(\mathrm{dd},{ }^{3} \mathrm{~J}_{\mathrm{HH}}=8.7\right.$ and $\left.1.5 \mathrm{~Hz}, 1 \mathrm{H}\right), 2.58\left(\mathrm{dd},{ }^{2} \mathrm{~J}_{\mathrm{HH}}=18.9 \mathrm{~Hz}\right.$ and ${ }^{3} \mathrm{~J}_{\mathrm{HH}}=$ $1.7 \mathrm{~Hz}, 1 \mathrm{H}), 0.90\left(\mathrm{t},{ }^{3} J_{\mathrm{HH}}=8.0 \mathrm{~Hz}, 3 \mathrm{H}\right), 0.55\left(\mathrm{q},{ }^{3} J_{\mathrm{HH}}=7.8 \mathrm{~Hz}, 2 \mathrm{H}\right), 0.02(\mathrm{~s}, 3 \mathrm{H}),-0.01$ (s, 3H). ${ }^{13} \mathrm{C} \mathrm{NMR}\left(\mathrm{CDCl}_{3}\right): \delta 204.6,152.3,143.0,138.4,132.3,119.5,107.4,101.5,40.1$, 24.0, 7.2, 5.8, -4.8, -5.2. HRMS (ESI) calcd for $\mathrm{C}_{14} \mathrm{H}_{19} \mathrm{O}_{3} \mathrm{Si}\left(\mathrm{M}+\mathrm{H}^{+}\right)$263.1098, found 263.1104 . 


\section{X-ray Crystal Structure of $\left[\mathrm{Rh}((R, R)-3)(\mathrm{MeCN})_{2}\right] \mathrm{PF}_{6}$}

\section{Data Collection}

A deep red dichloromethane solution of $\left[\mathrm{Rh}((R, R)-3)(\mathrm{MeCN})_{2}\right] \mathrm{PF}_{6}$ was prepared. Crystals suitable for X-ray analysis were obtained by layering of $\mathrm{Et}_{2} \mathrm{O}$ at room temperature.

A red prism crystal of $\mathrm{C}_{70} \mathrm{H}_{56} \mathrm{O}_{2} \mathrm{~F}_{6} \mathrm{~N}_{2} \mathrm{P}_{3} \mathrm{Rh}$ having approximate dimensions of 0.45 x $0.30 \times 0.30 \mathrm{~mm}$ was mounted on a glass fiber. All measurements were made on a Rigaku RAXIS RAPID imaging plate area detector with graphite monochromated Mo-K $\alpha$ radiation.

Indexing was performed from 6 oscillations that were exposed for 150 seconds. The crystal-to-detector distance was $127.40 \mathrm{~mm}$.

Cell constants and an orientation matrix for data collection corresponded to a primitive monoclinic cell with dimensions:

$$
\begin{aligned}
& \mathrm{a}=11.64(3) \AA \\
& \mathrm{b}=21.09(3) \AA \quad \AA=94.94(8)^{\circ} \\
& \mathrm{c}=11.95(2) \AA \\
& \mathrm{V}=2921(8) \AA^{3}
\end{aligned}
$$

For $\mathrm{Z}=2$ and F.W. $=1267.04$, the calculated density is $1.44 \mathrm{~g} / \mathrm{cm}^{3}$. Based on the systematic absences of:

$0 \mathrm{k} 0: \mathrm{k} \pm 2 \mathrm{n}$

packing considerations, a statistical analysis of intensity distribution, and the successful solution and refinement of the structure, the space group was determined to be:

$$
\mathrm{P} 2{ }_{1}(\# 4)
$$

The data were collected at a temperature of $-150 \pm 1^{\circ} \mathrm{C}$ to a maximum $2 \theta$ value of $54.9^{\circ}$. A total of 44 oscillation images were collected. A sweep of data was done using $\omega$ scans from 130.0 to $190.0^{\circ}$ in $5.0^{\circ}$ step, at $\chi=45.0^{\circ}$ and $\phi=30.0^{\circ}$. The exposure rate was $30.0\left[\mathrm{sec} . /^{\circ}\right.$ ]. A second sweep was performed using $\omega$ scans from 0.0 to 
$160.0^{\circ}$ in $5.0^{\circ}$ step, at $\chi=45.0^{\circ}$ and $\phi=180.0^{\circ}$. The exposure rate was 30.0 [sec. $/{ }^{\circ}$ ]. The crystal-to-detector distance was $127.40 \mathrm{~mm}$. Readout was performed in the $0.100 \mathrm{~mm}$ pixel mode.

\section{Data Reduction}

Of the 26569 reflections that were collected, 12810 were unique $\left(R_{i n t}=0.164\right)$.

The linear absorption coefficient, $\mu$, for Mo-K $\alpha$ radiation is $4.4 \mathrm{~cm}^{-1}$. The data were corrected for Lorentz and polarization effects.

\section{Structure Solution and Refinement}

The structure was solved by direct methods ${ }^{6}$ and expanded using Fourier techniques. ${ }^{7}$ The non-hydrogen atoms were refined anisotropically. Hydrogen atoms were refined using the riding model. The final cycle of full-matrix least-squares refinement ${ }^{8}$ on $\mathrm{F}^{2}$ was based on 12810 observed reflections and 758 variable parameters and converged (largest parameter shift was 0.00 times its esd) with unweighted and weighted agreement factors of:

$$
\begin{gathered}
\mathrm{R} 1=\Sigma|| \mathrm{Fo}|-| \mathrm{FC}|| / \Sigma|\mathrm{Fo}|=0.084 \\
\mathrm{wR} 2=\left[\Sigma\left(\mathrm{w}\left(\mathrm{Fo}^{2}-\mathrm{FC}^{2}\right)^{2}\right) / \Sigma \mathrm{w}\left(\mathrm{Fo}^{2}\right)^{2}\right]^{1 / 2}=0.232
\end{gathered}
$$

The standard deviation of an observation of unit weight ${ }^{9}$ was 0.85 . A Sheldrick weighting scheme was used. The maximum and minimum peaks on the final difference Fourier map corresponded to 1.41 and $-0.74 \mathrm{e}^{-} / \AA^{3}$, respectively.

Neutral atom scattering factors were taken from Cromer and Waber. ${ }^{10}$ Anomalous dispersion effects were included in Fcalc; ${ }^{11}$ the values for $\Delta \mathrm{f}^{\prime}$ and $\Delta \mathrm{f}^{\prime \prime}$

${ }^{6}$ SIR92: Altomare, A.; Cascarano, G.; Giacovazzo, C.; Guagliardi, A.; Burla, M.; Polidori, G.; Camalli, M. J. Appl. Cryst. 1994, 27, 435.

${ }^{7}$ DIRDIF99: Beurskens, P. T.; Admiraal, G.; Beurskens, G.; Bosman, W. P.; de Gelder, R.; Israel, R; Smits, J. M. M. The DIRDIF-99 program system, Technical Report of the Crystallography Laboratory, University of Nijmegen, The Netherlands (1999).

${ }^{8}$ Least Squares function minimized: (SHELXL97) $\Sigma w\left(\mathrm{~F}_{\mathrm{O}}^{2}-\mathrm{F}_{\mathrm{C}}{ }^{2}\right)^{2} \quad$ where $\mathrm{w}=$ Least Squares weights.

${ }^{9}$ Standard deviation of an observation of unit weight:

$\left[\Sigma w\left(\mathrm{~F}_{\mathrm{O}}^{2}-\mathrm{F}_{\mathrm{C}}{ }^{2}\right)^{2} /\left(\mathrm{N}_{\mathrm{O}}-\mathrm{N}_{\mathrm{V}}\right)\right]^{1 / 2}$ where: $\mathrm{N}_{\mathrm{O}}=$ number of observations, $\mathrm{N}_{\mathrm{V}}=$ number of variables 
were those of Creagh and McAuley. ${ }^{12}$ The values for the mass attenuation coefficients are those of Creagh and Hubbell. ${ }^{13}$ All calculations were performed using the CrystalStructure ${ }^{14,15}$ crystallographic software package except for refinement, which was performed using SHELXL-97. ${ }^{16}$

The crystal structure has been deposited at the Cambridge Crystallographic Data Centre (deposition number: CCDC 284625). The data can be obtained free of charge via the Internet at www.ccdc.cam.ac.uk/conts/retrieving.html.

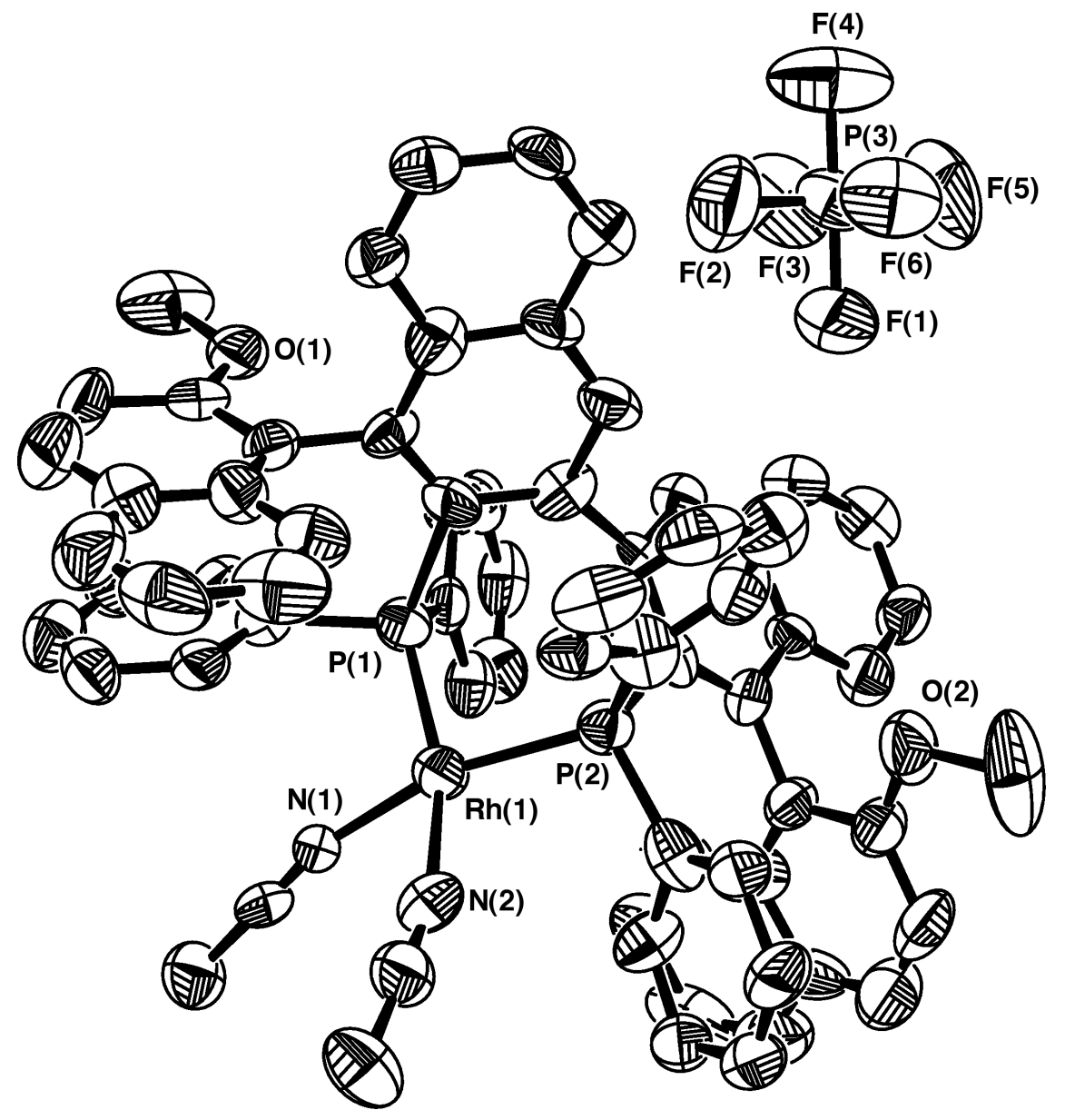

${ }^{10}$ Cromer, D. T.; Waber, J. T. "International Tables for X-ray Crystallography", Vol. IV, The Kynoch Press, Birmingham, England, Table 2.2 A (1974).

${ }^{11}$ Ibers, J. A.; Hamilton, W. C. Acta Crystallogr. 1964, 17, 781.

${ }^{12}$ Creagh, D. C.; McAuley, W. J . "International Tables for Crystallography", Vol C, (Wilson, A. J. C., ed.), Kluwer Academic Publishers, Boston, Table 4.2.6.8, pages 219-222 (1992).

${ }^{13}$ Creagh, D. C.; Hubbell, J. H. "International Tables for Crystallography", Vol C, (Wilson, A. J. C., ed.), Kluwer Academic Publishers, Boston, Table 4.2.4.3, pages 200-206 (1992).

${ }_{14}$ CrystalStructure 3.6.0: Crystal Structure Analysis Package, Rigaku and Rigaku/MSC (2000-2004). 9009 New Trails Dr. The Woodlands TX 77381 USA.

${ }^{15}$ CRYSTALS Issue 10: Watkin, D. J.; Prout, C. K.; Carruthers, J. R.; Betteridge, P. W. Chemical Crystallography Laboratory, Oxford, UK. (1996).

${ }^{16}$ SHELX97: Sheldrick, G. M. (1997). 
Experimental Details

\section{A. Crystal Data}

Empirical Formula

Formula Weight

Crystal Color, Habit

Crystal Dimensions

Crystal System

Lattice Type

Indexing Images

Detector Position

Pixel Size

Lattice Parameters

Space Group

$Z$ value

Dcalc

$\mathrm{F}_{000}$

$\mu(\operatorname{MoK} \alpha)$
$\mathrm{C}_{70} \mathrm{H}_{56} \mathrm{O}_{2} \mathrm{~F}_{6} \mathrm{~N}_{2} \mathrm{P}_{3} \mathrm{Rh}$

1267.04

red, prism

$0.45 \times 0.30 \times 0.30 \mathrm{~mm}$

monoclinic

Primitive

6 oscillations @ 150.0 seconds

$127.40 \mathrm{~mm}$

$0.100 \mathrm{~mm}$

$$
\begin{aligned}
& \mathrm{a}=11.64(3) \AA \\
& \mathrm{b}=21.09(3) \AA \\
& \mathrm{c}=11.95(2) \AA \\
& \beta=94.94(8)^{\circ} \\
& \mathrm{V}=2921(8) \AA^{3}
\end{aligned}
$$

$\mathrm{P} 2_{1}(\# 4)$

2

$1.440 \mathrm{~g} / \mathrm{cm}^{3}$

1300.00

$4.42 \mathrm{~cm}^{-1}$ 


\section{B. Intensity Measurements}

Diffractometer

Radiation

Detector Aperture

Data Images

$\omega$ oscillation Range $((\chi=45.0, \phi=30.0)$

Exposure Rate

$\omega$ oscillation Range $(\chi=45.0, \phi=180.0)$

Exposure Rate

Detector Position

Pixel Size

$2 \theta_{\max }$

No. of Reflections Measured

Corrections
Rigaku RAXIS-RAPID

$\operatorname{MoK} \alpha(\lambda=0.71075 \AA)$

graphite monochromated

$280 \mathrm{~mm} \times 256 \mathrm{~mm}$

44 exposures

$130.0-190.0^{\circ}$

$30.0 \mathrm{sec} . /^{\circ}$

$0.0-160.0^{\circ}$

$30.0 \mathrm{sec} . /^{\circ}$

$127.40 \mathrm{~mm}$

$0.100 \mathrm{~mm}$

$54.9^{\circ}$

Total: 26569

Unique: $26540(\operatorname{Rint}=0.164)$

Lorentz-polarization 


\section{Structure Solution and Refinement}

Structure Solution

Refinement

Function Minimized

Least Squares Weights

$2 \theta_{\max }$ cutoff

Anomalous Dispersion

No. Observations (All reflections)

No. Variables

Reflection/Parameter Ratio

Residuals: R (All reflections)

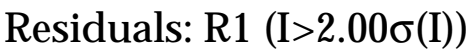

Residuals: wR2 (All reflections)

Goodness of Fit Indicator

Max Shift/Error in Final Cycle

Maximum peak in Final Diff. Map

Minimum peak in Final Diff. Map
Direct Methods (SIR92)

Full-matrix least-squares on $\mathrm{F}^{2}$

$\Sigma \mathrm{w}\left(\mathrm{Fo}^{2}-\mathrm{FC}^{2}\right)^{2}$

$\mathrm{w}=1 /\left[\sigma^{2}\left(\mathrm{Fo}^{2}\right)+(0.1000 \cdot \mathrm{P})^{2}\right.$

$+0.0000 \cdot \mathrm{P}$ ]

where $\mathrm{P}=\left(\operatorname{Max}\left(\mathrm{Fo}^{2}, 0\right)+2 \mathrm{Fc}^{2}\right) / 3$

$54.9^{\circ}$

All non-hydrogen atoms

12810

758

16.90

0.201

0.084

0.232

0.847

0.001

$1.41 \mathrm{e}^{-} / \AA^{3}$

$-0.74 \mathrm{e}^{-} / \AA^{3}$ 